DESY 02-107

OUTP-02-36-P

\title{
A Bound on Neutrino Masses From Baryogenesis
}

\author{
W. Buchmüller, P. Di Bari \\ Deutsches Elektronen-Synchrotron DESY, 22603 Hamburg, Germany \\ M. Plümacher \\ Theoretical Physics, University of Oxford, 1 Keble Road, \\ Oxford, OX1 3NP, United Kingdom
}

\begin{abstract}
Properties of neutrinos, the lightest of all elementary particles, may be the origin of the entire matter-antimatter asymmetry of the universe. This requires that neutrinos are Majorana particles, which are equal to their antiparticles, and that their masses are sufficiently small. Leptogenesis, the theory explaining the cosmic matterantimatter asymmetry, predicts that all neutrino masses are smaller than $0.2 \mathrm{eV}$, which will be tested by forthcoming laboratory experiments and by cosmology.
\end{abstract}


Neutrinos are unique elementary particles. They have only weak interactions and can therefore travel astronomically large distances without being absorbed. In particular, they reach the earth from the center of the sun. The study of solar and atmospheric neutrinos, produced by cosmic rays in the earth's atmosphere, has provided evidence for neutrino oscillations. These take place between the three species of neutrinos, $\nu_{e}, \nu_{\mu}$ and $\nu_{\tau}$, which belong to the three families of elementary particles with the charged leptons electron $(e)$, muon $(\mu)$ and tau $(\tau)$, respectively.

Neutrinos are electrically neutral, spin- $\frac{1}{2}$ particles. In the standard model of strong and electroweak interactions neutrinos are massless. As a consequence, electron-, muon-, and tau-number are conserved. Due to the evidence for neutrino oscillations we now know that neutrinos have mass and that the three lepton numbers are not separately conserved. However, we still do not know whether the total lepton number, $L=L_{e}+L_{\mu}+L_{\tau}$, is also violated. In this case neutrinos would be Majorana particles which are equal to their antiparticles.

The standard model of particle physics can be derived as low energy effective theory from a more fundamental grand unified theory (GUT), whose symmetry becomes manifest only at much shorter distances, or correspondingly larger energies. The simplest GUT which unites the strong and electroweak forces, and where all quarks and leptons can turn into each other via gauge interactions, is based on the group $\mathrm{SO}(10)$ [1]. This theory predicts the existence of 'sterile' neutrinos $\nu_{R}$, one for each quark-lepton generation, which have no strong or electroweak interactions, unlike all other known elementary particles. After the breaking of the GUT group $\mathrm{SO}(10)$ to the standard model gauge group $\mathrm{SU}(3) \times \mathrm{SU}(2) \times \mathrm{U}(1)$, the sterile neutrinos become massive Majorana fermions, $N=\nu_{R}+\nu_{R}^{c}$, where the superscript 'c' denotes antiparticles.

Heavy Majorana neutrinos with masses $M$ of order the GUT mass scale, $\mathrm{E}_{G} \sim$ $10^{15} \mathrm{GeV}$, also give rise to small neutrino masses through mixing, which is the so-called seesaw mechanism [2]. The light neutrino mass eigenstates $\nu_{1}, \nu_{2}$ and $\nu_{3}$ are mixtures of $\nu_{e}, \nu_{\mu}$ and $\nu_{\tau}$, and again Majorana neutrinos, i.e. identical to their antiparticles, $\nu_{i}=\nu_{i}^{c}$. Assuming for the neutrino masses the ordering $m_{1}<m_{2}<m_{3}$, one expects for the largest mass $m_{3}$ as consequence of the seesaw mechanism,

$$
m_{3} \sim \frac{v^{2}}{M} \sim 0.01 \mathrm{eV}
$$

where we have chosen for $v$ the mass scale of electroweak symmetry breaking of order the W-boson mass, $v \simeq 174 \mathrm{GeV}$.

The evidence for neutrino oscillations reported by the Super-Kamiokande [3] and SNO [4] collaborations suggests $\nu_{\mu} \rightarrow \nu_{\tau}$ and $\nu_{e} \rightarrow \nu_{\mu}$ oscillations. The analysis yields two 
neutrino mass differences, $\Delta m_{\text {atm }}^{2}=(1.6-3.9) \times 10^{-3} \mathrm{eV}^{2}[3]$ and $\Delta m_{\text {sol }}^{2}=(0.2-2) \times$ $10^{-4} \mathrm{eV}^{2}$ [1]. It is very remarkable that $\sqrt{\Delta m_{\text {atm }}^{2}} \sim 0.05 \mathrm{eV}$ and $\sqrt{\Delta m_{\text {sol }}^{2}} \sim 0.008 \mathrm{eV}$ are consistent with the estimate (11). This suggests that the extrapolation to the GUT mass scale $\mathrm{E}_{G} \sim 10^{15} \mathrm{GeV}$ by means of the seesaw mass relation may indeed be correct!

So far, however, we do not know the absolute scale of neutrino masses since oscillation experiments can only determine differences of mass squares. An upper bound on a weighted sum of neutrino masses has been obtained from a study of the electron energy spectrum in tritium $\beta$-decay, $m_{\nu_{e}}=\left(\sum_{i}\left|U_{e i}\right|^{2} m_{i}^{2}\right)^{1 / 2}<2.2 \mathrm{eV}$ [0]. Here $U_{e i}(i=1 \ldots 3)$ are elements of the 'leptonic mixing matrix' which determine the couplings of an electron to a $\mathrm{W}$-boson and the neutrino mass eigenstates $\nu_{i}$. An even more stringent bound has been obtained from neutrinoless double $\beta$-decay, which for a nucleus with $Z$ protons and $N$ neutrons corresponds to the transition $A(Z, N) \rightarrow A(Z+2, N-2)+e^{-}+e^{-}$. Note, that this process violates lepton number! Non-observation of neutrinoless double $\beta$-decay yields an upper bound on the effective neutrino mass $m_{e e}=\left|\sum_{i} U_{e i}^{2} m_{i}\right|<0.38 \kappa \mathrm{eV}$, where $\kappa=\mathcal{O}(1)$ represent the uncertainty of the nuclear matrix element [6] .

Important information on neutrino masses also comes from cosmology. The requirement that neutrinos do not overclose the universe yields the limit $\sum_{i} m_{\nu_{i}}<40 \mathrm{eV}$. A much stronger bound has recently been obtained from the 2dF Galaxy Redshift Survey, $\sum_{i} m_{\nu_{i}}<1.8 \mathrm{eV}$ at $95 \%$ confidence level [7], which is comparable to the bound from tritium $\beta$-decay.

Baryons, i.e. protons and neutrons, dominate by far the cosmic mass fraction of visible matter. As far as we know, the universe contains no primordial antimatter. Hence, the baryon density $n_{B}$ is equal to the difference of baryon and antibaryon densities, which is conventionally normalized to the photon density, $\eta_{B}=\left(n_{B}-n_{\bar{B}}\right) / n_{\gamma}$. The precision of measurements of this baryon asymmetry has significantly improved with the observation of the acoustic peaks in the cosmic microwave background radiation (CMB). The BOOMERanG and DASI experiments have measured the baryon asymmetry with a $(1 \sigma)$ standard error of $\sim 15 \%$ [8, 9],

$$
\eta_{B 0}^{C M B}=\left(6.0_{-0.8}^{+1.1}\right) \times 10^{-10}
$$

which is consistent with the result of standard primordial nucleosynthesis (BBN), obtained from the measurement of relic nuclear abundances [10].

At first sight, it may appear very surprizing that the observed cosmological baryon asymmetry should be related to neutrino properties. The reason is a deep connection [11] between baryon and lepton number in the standard model of particle physics which is hidden today, but which becomes visible at very high temperatures above the scale of 
electroweak symmetry breaking, $T>v \sim 100 \mathrm{GeV} \sim 10^{15} \mathrm{~K}$. At such high temperatures, which may have been realized in the early universe, quarks and leptons are rapidly transformed into each other by so-called sphaleron processes [12], and lepton and baryon number are no longer separately conserved. In thermal equilibrium baryon and lepton asymmetries have a constant ratio determined by the standard model.

A baryon asymmetry can be dynamically generated in an expanding universe if the particle interactions violate baryon number, charge conjugation $(C)$ and joint parity and charge conjugation $(C P)$, and if the evolution of the universe leads to a departure from thermal equilibrium [13]. All these conditions can be fulfilled in decays of heavy Majorana neutrinos $(N)$ into light leptons $(l)$ and Higgs bosons $(\phi) . C P$ violating, out-of-equilibrium decays $N \rightarrow l \phi$ generate naturally a lepton asymmetry which, by means of the sphaleron processes, is partially transformed into a baryon asymmetry. This is the simple and elegant leptogenesis mechanism [14].

The non-equilibrium process of baryogenesis is described by means of Boltzmann equations. The final baryon asymmetry is the result of a competition between production processes and washout processes which tend to erase any generated asymmetry. We shall assume that the dominant contribution is given by decays of $N_{1}$, the lightest of the heavy Majorana neutrinos. This assumption is well justified in the case of a mass hierarchy among the heavy neutrinos, i.e. $M_{1} \ll M_{2}, M_{3}$, and it is also known to be a good approximation, if $M_{2,3}-M_{1}=\mathcal{O}\left(M_{1}\right)$. The case of partial degeneray, $M_{2,3}-M_{1} \ll M_{1}$, requires a special treatment. In a recent detailed study [15] it has been shown that the final baryon asymmetry depends on just four parameters: the mass $M_{1}$ of $N_{1}$, the lightest of the heavy Majorana neutrinos; the $C P$ asymmetry in $N_{1}$ decays, $\varepsilon_{1}=\left(\Gamma\left(N_{1} \rightarrow l \phi\right)-\Gamma\left(N_{1} \rightarrow l^{c} \phi^{c}\right)\right) /\left(\Gamma\left(N_{1} \rightarrow l \phi\right)+\Gamma\left(N_{1} \rightarrow l^{c} \phi^{c}\right)\right)$; the effective neutrino mass $\widetilde{m}_{1}$, with $m_{1} \leq \widetilde{m}_{1} \lesssim m_{3}$, which is a measure of the strength of the coupling of $N_{1}$ to the thermal bath, and, finally, the sum of all neutrino masses squared, $\bar{m}^{2}=m_{1}^{2}+m_{2}^{2}+m_{3}^{2}$, which controls an important class of washout processes. Together with the two mass squared differences $\Delta m_{\text {atm }}^{2}$ and $\Delta m_{\text {sol }}^{2}$, the sum $\bar{m}^{2}$ determines all neutrino masses.

The CP asymmetry $\varepsilon_{1}$ satisfies an upper bound, $\left|\varepsilon_{1}\right|<\varepsilon\left(M_{1}, \bar{m}\right)$ [16]. The maximal baryon asymmetry produced by $N_{1}$ decays can then be expressed in terms of a maximal $C P$ asymmetry $\varepsilon$ and an efficiency factor $\kappa_{0}$ [15],

$$
\eta_{B 0}^{\max }\left(\widetilde{m}_{1}, M_{1}, \bar{m}\right)=0.96 \times 10^{-2} \varepsilon\left(M_{1}, \bar{m}\right) \kappa_{0}\left(\widetilde{m}_{1}, M_{1}, \bar{m}\right)
$$

with

$$
\varepsilon\left(M_{1}, \bar{m}\right)=\frac{3}{16 \pi} \frac{M_{1}}{v^{2}} \frac{\Delta m_{a t m}^{2}+\Delta m_{s o l}^{2}}{m_{3}} .
$$




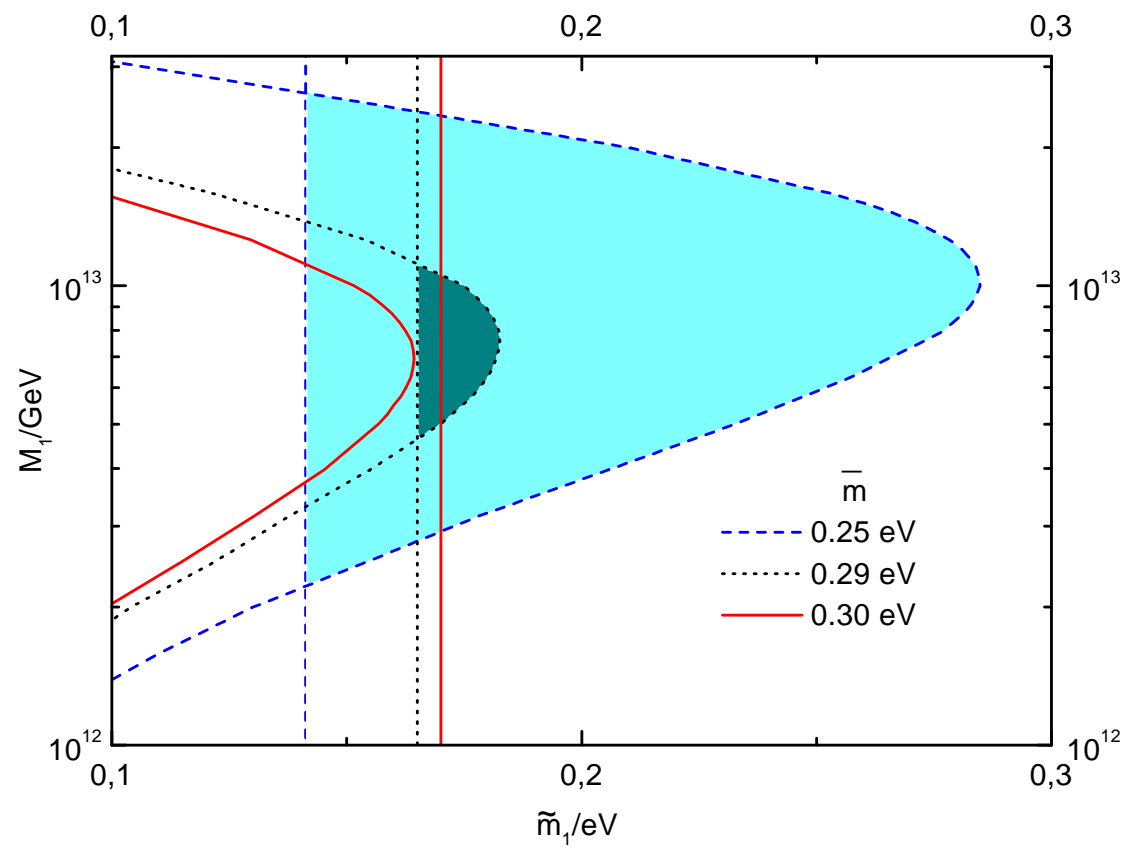

Figure 1: Regions in the $\widetilde{m}_{1}-M_{1}$-plane allowed by baryogenesis for different values of $\bar{m}$. The boundaries are defined by $\eta_{B 0}^{\max }\left(\widetilde{m}_{1}, M_{1}, \bar{m}\right)=\eta_{B 0}^{C M B}$ and by $\widetilde{m}_{1}=m_{1}(\bar{m})$.

Note, that the maximal $C P$ asymmetry is independent of the mass hierarchy among the neutrinos. For a 'normal' hierarchy, i.e. $\Delta m_{a t m}^{2}=m_{3}^{2}-m_{2}^{2}>m_{2}^{2}-m_{1}^{2}=\Delta m_{\text {sol }}^{2}$, the individual neutrino masses are given by

$$
\begin{aligned}
& m_{3}^{2}=\frac{1}{3}\left(\bar{m}^{2}+2 \Delta m_{a t m}^{2}+\Delta m_{\text {sol }}^{2}\right) \\
& m_{2}^{2}=\frac{1}{3}\left(\bar{m}^{2}-\Delta m_{a t m}^{2}+\Delta m_{\text {sol }}^{2}\right) \\
& m_{1}^{2}=\frac{1}{3}\left(\bar{m}^{2}-\Delta m_{\text {atm }}^{2}-2 \Delta m_{\text {sol }}^{2}\right) .
\end{aligned}
$$

Inserting the expression for $m_{3}$ into eq. (田) yields the maximal asymmetry $\varepsilon$ as function of $M_{1}$ and $\bar{m}$. Analogous formulae hold in the case of an 'inverse' hierarchy where $\Delta m_{\text {sol }}^{2}=$ $m_{3}^{2}-m_{2}^{2}<m_{2}^{2}-m_{1}^{2}=\Delta m_{\text {atm }}^{2}$. The difference between 'normal' and 'inverted' hierarchy is irrelevant for the following analysis.

If leptogenesis is the true mechanism of baryogenesis, the maximal baryon asymmetry has to be larger than the observed one, for which we choose the most precise CMB value (2), i.e. we require $\eta_{B 0}^{\max }\left(\widetilde{m}_{1}, M_{1}, \bar{m}\right) \gtrsim \eta_{B 0}^{C M B}$. This condition imposes an important restriction on the space of neutrino parameters $\widetilde{m}_{1}, M_{1}$ and $\bar{m}$. Since the strength of 


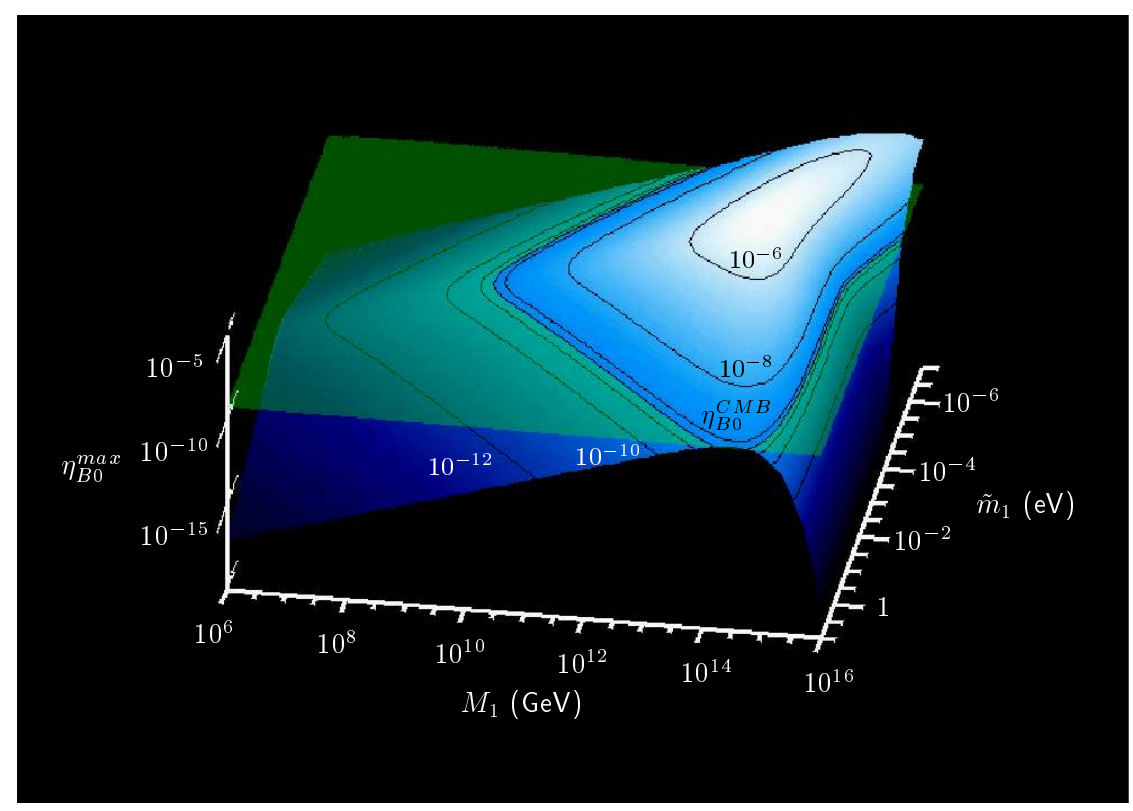

Figure 2: Maximal baryon asymmetry $\eta_{B 0}^{\max }$ (blue) as function of $\widetilde{m}_{1}$ and $M_{1}$ for $\bar{m}=$ $0.05 \mathrm{eV}$. The black lines are curves of constant baryon asymmetry with the value indicated. The lines around the intersection with the green plane correspond to the measured value and the upper/lower limits at $3 \sigma$.

washout processes increases with increasing $\bar{m}$, one obtains in particular an upper bound on $\bar{m}$.

In order to determine the upper bound on $\bar{m}$ one has to calculate the efficiency factor $\kappa_{0}$ by solving the Boltzmann equations for different points in the neutrino parameter space $\left(\widetilde{m}_{1}, M_{1}, \bar{m}\right)$. Results of a numerical scan are displayed in fig. 1 which shows the curves defined by $\eta_{B 0}^{\max }\left(\widetilde{m}_{1}, M_{1}, \bar{m}\right)=\eta_{B 0}^{C M B}$ and by $\widetilde{m}_{1}=m_{1}(\bar{m})$, for $\bar{m}=0.25 \mathrm{eV}$ (dashed), $0.29 \mathrm{eV}$ (dotted) and $0.30 \mathrm{eV}$ (full). For the two smaller values of $\bar{m}$ domains exist which allow large enough baryon asymmetry. For $\bar{m}=0.30 \mathrm{eV}$ successfull baryogenesis is no longer possible.

The determination of a theoretical error on the upper bound $\bar{m}<0.30 \mathrm{eV}$ would require a systematic quantitative treatment of baryogenesis, which goes beyond the Boltzmann equations. This is not yet available. However, all corrections we are aware of tend to decrease the generated baryon asymmetry. Including these corrections would therefore lead to an even stronger upper bound on $\bar{m}$. Based on the studies in [17], we expect that in the supersymmetric standard model the bound on $\bar{m}$ is rather similar to the non-supersymmetric case. A detailed numerical investigation is in progress.

For the upper bound $\bar{m}^{\max }=0.30 \mathrm{eV}$ the individual neutrino masses are $m_{3}=0.18 \mathrm{eV}$ and $m_{1}=m_{2}=0.17 \mathrm{eV}$. Using the unitarity of the leptonic mixing matrix, $\sum_{i}\left|U_{e i}\right|^{2}=1$, 
one then obtains for the mass measured in tritum $\beta$-decay, $m_{\nu_{e}}<0.18 \mathrm{eV}$. This value is smaller than the sensitivity aimed at by the KATRIN collaboration [18]. The same bound as for $m_{\nu_{e}}$ one also finds for the Majorana mass measued in neutrinoless double $\beta$-decay, $m_{e e}<0.18 \mathrm{eV}$. This prediction can be tested by the GENIUS project [19]. Cosmological bounds on neutrino masses will also improve, and the Sloan Digital Sky Survey combined with CMB data from the MAP experiment is expected to reach the sensitivity 20 of the leptogenesis bound, $\sum_{i} m_{i} \leq 0.52 \mathrm{eV}$.

For strongly hierarchical neutrinos, $m_{1}<m_{2} \ll m_{3} \simeq \bar{m} \simeq 0.05 \mathrm{eV}$, successful baryogenesis is possible for a large domain in the $\widetilde{m}_{1}-M_{1}$-plane. This can be seen in fig. 2 where the surface $\eta_{B 0}^{\max }$ is shown for $\bar{m}=0.05 \mathrm{eV}$. The intersection with the plane $\eta_{B 0}^{\mathrm{CMB}}$ yields the allowed region of parameters. For the heavy neutrino mass one obtains the lower bound $M_{1}>4 \times 10^{8} \mathrm{GeV}$ [15]. In many models of neutrino masses the $C P$ asymmetry saturates the upper bound, $\left|\varepsilon_{1}\right| \sim\left|\varepsilon_{1}^{\max }\right|$ (cf. [15]). The CMB baryon asymmetry then yields a precise relation between $\widetilde{m}_{1}$ and $M_{1}$. For the particularly interesting range $0.001 \mathrm{eV} \lesssim \widetilde{m}_{1} \lesssim 0.1 \mathrm{eV}$ one has the simple relation $M_{1} \simeq 3 \times 10^{10} \mathrm{GeV} \widetilde{m}_{1} /(0.01 \mathrm{eV})$. Such values of $M_{1}$ are naturally obtained in $\mathrm{SO}(10)$ grand unified models where lepton number is broken at the GUT mass scale $\mathrm{E}_{G} \sim 10^{15} \mathrm{GeV}$. This is the scenario studied in [21], with a baryogenesis temperature $T_{B} \sim M_{1} \sim 10^{10} \mathrm{GeV}$. Such a high temperature has important implications for the dark matter problem due to the abundance of thermally produced gravitinos.

In summary, decays of heavy Majorana neutrinos provide a simple and elegant explanation of the origin of matter in our universe. Before recombination and primordial nucleosynthesis, baryogenesis would then be the next important period in the early universe, at a temperature $T_{B} \sim 10^{10} \mathrm{GeV}$ and a corresponding time $t_{B} \sim 10^{-26} \mathrm{~s}$. An unequivocal prediction of this picture is that neutrinos are Majorana particles with masses below $0.2 \mathrm{eV}$, which will be tested by forthcoming laboratory experiments and by cosmology.

\section{Acknowledgment}

M.P. was supported by the EU network "Supersymmetry and the Early Universe" under contract no. HPRN-CT-2000-00152.

\section{References}

[1] H. Georgi, in Particles and Fields, ed. C. E. Carlson (AIP, NY, 1975) p. 575;

H. Fritzsch, P. Minkowski, Ann. of Phys. 93 (1975) 193 
[2] T. Yanagida, in Workshop on Unified Theories, KEK report 79-18 (1979) p. 95;

M. Gell-Mann, P. Ramond, R. Slansky, in Supergravity (North Holland, Amsterdam, 1979) eds. P. van Nieuwenhuizen, D. Freedman, p. 315

[3] Super-Kamiokande Collaboration, T. Toshito et al., hep-ex/0105023

[4] SNO Collaboration, Q. R. Ahmad et al., nucl-ex/0204008; nucl-ex/0204009

[5] Mainz Collaboration, J. Bonn et al., Nucl. Phys. Proc. Suppl. 91 (2001) 273

[6] Heidelberg-Moscow Collaboration, H. V. Klapdor-Kleingrothaus et al., Eur. Phys. J. A12 (2001) 147

[7] O. Elgaroy et al., astro-ph/0204152

[8] P. de Bernardis et al., Astrophys. J. 564 (2002) 559

[9] C. Pryke et al., Astrophys. J. 568 (2002) 46

[10] B. D. Fields, S. Sarkar, in Review of Particle Physics, Phys. Rev. D 66 (2002) 010001

[11] G. 't Hooft, Phys. Rev. Lett. 37 (1976) 8

[12] V. A. Kuzmin, V. A. Rubakov, M. E. Shaposhnikov, Phys. Lett. B 155 (1985) 36

[13] A. D. Sakharov, JETP Lett. 5 (1967) 24

[14] M. Fukugita, T. Yanagida, Phys. Lett. B 174 (1986) 45

[15] W. Buchmüller, P. Di Bari, M. Plümacher, hep-ph/0205349

[16] S. Davidson, A. Ibarra, Phys. Lett. B 535 (2002) 25

[17] M. Plümacher, Nucl. Phys. B 530 (1998) 207

[18] KATRIN Collaboration, A. Osipowicz et al., hep-ex/0109033

[19] H. V. Klapdor-Kleingrothaus, Nucl. Phys. Proc. Suppl. 100 (2001) 350

[20] W. Hu, D. J. Eisenstein, M. Tegmark, Phys. Rev. Lett. 80 (1998) 5255

[21] W. Buchmüller, M. Plümacher, Phys. Lett. B 389 (1996) 73 